\title{
Preserved Sensitivity to Outcome Value after Lesions of the Basolateral Amygdala
}

\author{
Pam Blundell, ${ }^{1}$ Geoffrey Hall, ${ }^{1}$ and Simon Killcross ${ }^{2}$ \\ ${ }^{1}$ Department of Psychology, University of York, Heslington, York Y010 5DD, United Kingdom, and ${ }^{2}$ School of Psychology, Cardiff University, Cardiff CF10 \\ 3YG, United Kingdom
}

Recent work (Blundell et al., 2001; Balleine et al., 2003) has suggested that the basolateral region of the amygdala (BLA) is important in the representation of the sensory and incentive aspects of motivationally significant events. In common with other theories of function of the BLA, this predicts that lesions of the BLA will interfere with reinforcer devaluation after appetitive Pavlovian or instrumental conditioning. However, this hypothesis also predicts that BLA lesions will be without effect on postconditioning changes in reinforcer value if initial learning is only about the sensory aspects of otherwise neutral events. This interpretation is supported by evidence for significant detrimental effects of BLA lesions on reinforcer devaluation in a Pavlovian autoshaping procedure, but no effect of postconditioning devaluation using a sensory preconditioning procedure. These results demonstrate that animals with BLA lesions can remain sensitive to post-training changes in the motivational value of outcomes.

Key words: appetitive conditioning; basolateral amygdala; reward; sensory preconditioning; Pavlovian; instrumental; devaluation

\section{Introduction}

Experiments in rats have demonstrated that damage to the basolateral region of the amygdala (BLA) impairs the ability of animals to respond to post-training changes in the value of instrumental or Pavlovian rewards (Hatfield et al., 1996; Balleine et al., 2003). Although appetitive Pavlovian conditioning because of pairings of a conditioned stimulus (CS) with a food unconditioned stimulus (US) proceeds normally in rats with BLA lesions, subsequent reward devaluation brought about by pairing the US with nausea-inducing injections of lithium chloride ( $\mathrm{LiCl}$ ) fails to reduce magazine approach conditioned responses (CRs) in the presence of the CS. This effect is not because of a failure of the reward devaluation, because consumption tests demonstrate intact taste aversion in lesioned animals that is of similar magnitude to that observed in sham-operated control animals. Hatfield et al. (1996) concluded that the BLA was involved in associative processes that give CS access to the motivational value of their associated US, and BLA lesions abolished the ability of rats to "spontaneously adjust CRs to post training alterations in the value of the US" (p 5261).

After a recent study (Blundell et al., 2001), we suggested a more specific interpretation of BLA function (Killcross and Blundell, 2002; Balleine et al., 2003). In brief, we demonstrated that BLA lesions did not disrupt acquisition of an instrumental conditional discrimination but did disrupt the ability of rats to use different reward types to enhance the rate of acquisition. Furthermore, in a Pavlovianinstrumental transfer study, rats were clearly able to show a motivational influence of prior CS-US associations but were unable to

\footnotetext{
Received March 17, 2003; revised June 30, 2003; accepted July 2, 2003.

This work was supported by a United Kingdom Medical Research Center (MRC) Career Establishment Grant to S.K. and by an MRC studentship to P.B.

Correspondence should be addressed to Dr. Pam Blundell, Department of Psychology, University of York, Heslington, York Y010 5DD, UK. E-mail: p.blundell@psych.york.ac.uk.

Copyright $\odot 2003$ Society for Neuroscience $\quad$ 0270-6474/03/237702-08\$15.00/0
}

show any reinforcer specificity in these associations. We proposed that BLA lesions disrupted the use of CS-evoked representations of the unique sensory or hedonic aspects of motivationally significant events, but not the more general reinforcing aspects. A prediction of this interpretation is that under certain circumstances, BLA-lesioned animals will retain sensitivity to changes in the motivational value of associative outcomes. One such scenario is sensory preconditioning. In this procedure, initially neutral stimuli (S1 and S2) are paired together, after which S2 is paired with a motivationally significant event. At test, animals show change responding to S1 because of the newly acquired motivational value of S2 (Rizley and Rescorla, 1972). Because animals with BLA lesions are not impaired in the basic acquisition of taste aversions (Dunn and Everitt, 1988; but see Morris et al., 1999) and they should not be impaired in the acquisition of associations between the sensory properties of neutral stimuli, then they should also show sensitivity to post-training alterations to the motivational significance of S2. Evidence that they do would provide support for the claim that animals with BLA lesions can indeed learn about the current motivational value of associatively activated outcome representations.

Experiment 1 sought to confirm that BLA lesions do not produce deficits in appetitive Pavlovian conditioning but do impair sensitivity to post-training reward devaluation, using a lever approach autoshaping procedure. Experiment 2 examined the sensitivity of BLA-lesioned animals to reward devaluation using a sensory preconditioning procedure.

\section{Materials and Methods \\ Experiment 1: BLA lesions and devaluation of autoshaped responding}

Subjects

We used 16 naive, male, hooded Lister rats. Before surgery, their mean ad libitum weight was $275 \mathrm{gm}$ (range, 250-285). The rats were housed in pairs in a climate-controlled vivarium (lights on 8 A.M. to 8 P.M.). 
Subjects were tested during the light portion of the cycle. Before the start of training, the rats were reduced to $80 \%$ of their ad libitum postsurgical recovery weights. After completion of behavioral testing, the rats were killed, and their brains removed for histological analysis.

\section{Surgery}

Under anesthesia, eight rats received bilateral lesions of the BLA produced by injection of quinolinic acid $(0.09 \mathrm{M})$; the remainder received a control surgical procedure. Anesthesia was induced by an intraperitoneal injection $(10 \mathrm{ml} / \mathrm{kg}$ ) of Avertin (made up as $1.25 \mathrm{ml}$ of Avertin concentrate added to $5 \mathrm{ml}$ of absolute alcohol and $62.5 \mathrm{ml}$ of physiological saline; Avertin concentrate consists of 100 gm of 2-2-2-tri-bromo-ethanol dissolved in $62 \mathrm{ml}$ of tertiary amyl alcohol). Additional injections ( $1 \mathrm{ml}$, i.p.) were given, if necessary. When a rat was fully anesthetized, it was placed in a stereotaxic frame (Stoelting Inc., Kiel, WI). The depth of anesthesia was monitored by assessing the pedal withdrawal reflex and responsivity to a mild tail pinch. An incision was made along the skull, and then skin and fascia were cleared to reveal bregma. A drill mounted on the stereotaxic frame was used to make burr holes above the injection sites. Injections (two on each side) were made with a 30 gauge needle attached by polythene tubing to a $1 \mu$ l syringe, which was controlled by an infusion pump (Harvard Apparatus, Holliston, MA). We made $0.25 \mu$ injections at the following coordinates: lateral, $\pm 4.6 \mathrm{~mm}$; anterior-posterior, -2.3 $\mathrm{mm},-3 \mathrm{~mm}$; ventral (from dura), $-7.3 \mathrm{~mm}$. Each injection was made over $2.5 \mathrm{~min}$, and the injection needle was left in place for an additional $2.5 \mathrm{~min}$ to allow the neurotoxin to diffuse. The skin was then closed with suture. Animals were then given an injection of saline if they were dehydrated. Animals were observed during recovery from anesthesia and were returned to the vivarium after starting to eat and drink. Animals were allowed to recover for at least 1 week with ad libitum access to food and water. The control surgical procedure was identical to that which was used to produce lesions, the only difference being that no quinolinic acid was injected.

\section{Histology}

After the completion of behavioral testing, animals were anesthetized with a lethal overdose of sodium pentobarbitol (Sagital; $2 \mathrm{ml}$ per animal, i.p.) and perfused via the ascending aorta with $0.1 \mathrm{M}$ PBS, $\mathrm{pH} 7.4$, followed by $4 \%$ paraformaldehyde. The brains were then removed and postfixed in $4 \%$ paraformaldehyde solution before being transferred into $20 \%$ sucrose solution. After $24 \mathrm{hr}$, the brains were frozen on a freezing microtome fast-freeze plate before coronal sections $(40 \mu \mathrm{m})$ were cut on a freezing microtome throughout the full extent of the lesioned area. Every fourth section was taken and mounted on a gelatin-coated glass slide and then stained for analysis using cresyl violet. Slides were coverslipped, dried, and then examined under microscope to assess the extent and nature of excitotoxin-induced neuronal damage. Areas of neuronal loss were mapped onto standardized sections of the rat brain (Swanson, 1998).

\section{Apparatus}

Behavioral procedures were performed in eight identical operant chambers ( $25 \mathrm{~cm}$ wide $\times 25 \mathrm{~cm}$ deep $\times 22 \mathrm{~cm}$ high; Paul Fray Ltd., Cambridge, UK), housed in light- and sound-attenuating boxes. Each chamber was fitted with two retractable levers, one located on each side of a central, recessed magazine that provided access, via a hinged Plexiglas flap, to a food magazine into which food pellets ( $45 \mathrm{mg}$ Formula A/I; P.J. Noyes, Lancaster, NH) could be delivered. In addition, each chamber was equipped with a peristaltic pump that, when operated for $0.5 \mathrm{sec}$, delivered $0.5 \mathrm{ml}$ of a $20 \%$ sucrose solution into the same magazine. Opening the magazine flap operated a micro-switch that provided a measure of approach to the site of food delivery. The floor of the chambers consisted of $185 \mathrm{~mm}$ diameter steel rods spaced $1.5 \mathrm{~cm}$ apart, perpendicular to the front wall of the chamber. Each chamber was illuminated by a single, 4.2 W house light located in the center of the ceiling. A BBC Master 128 microcomputer, equipped with a SPIDER extension for on-line control (Paul Fray Ltd.) controlled the equipment and recorded the data.

\section{Procedure}

Training. After recovery from surgery, each animal was assigned to one of the eight operant chambers and thereafter was always trained in that chamber. At the start of each session, the house light came on and remained on throughout the session. The house light remained on at the end of each session. The reinforcers used were $45 \mathrm{gm}$ Noyes food pellets and $0.5 \mathrm{ml}$ of $20 \% \mathrm{w} / \mathrm{v}$ sucrose solution. Pilot studies indicted that in normal rats these two rewards produce very similar levels of performance of conditioned behaviors such as magazine approach and lever pressing and are well matched for their motivational value. Training consisted of two stages - magazine training and autoshaped lever pressing. There were then several test sessions, for which the rewards were first devalued by specific satiety. One session was run on each day.

Magazine training. All of the rats were trained to collect food rewards during two 40 min magazine training sessions. Half the animals (equal numbers of sham- and BLA-lesioned animals) were trained to collect food pellets in the first training session, and half to collect sucrose solution. The following day, rats were trained to collect the alternative reward. The rewards were delivered on a random time $60 \mathrm{sec}$ schedule.

Autoshaped lever press training. In each session, the rats were given 20 $10 \mathrm{sec}$ presentations of a lever on a variable time schedule of $90 \mathrm{sec}$ (range, $80-100)$. During the last second of the lever presentation, a reinforcer, either a food pellet or sucrose solution, was delivered into the food magazine. There was no requirement for the animal to make any response to receive food reward. In each session, only one lever and one reward type was used. The rats were given training on each lever in successive sessions. The first eight sessions were of one type, and the next eight sessions were of the other type. There followed an additional 16 sessions of training with the sucrose reinforcer and 8 sessions of training with the pellet reinforcer. Over the first part of this phase of training, the two types of session were presented in alternation. It became apparent, however, that responding was developing more readily with pellets than with sucrose. In an attempt to achieve comparable levels of performance with the two reinforcers, eight extra sessions of training with sucrose were inserted into the alternating sequence toward the end of training. Whether it was the left or right lever that predicted sucrose solution was counterbalanced across animals, such that for half of the animals in each group, the left lever signaled sucrose, and for the other half, it signaled food pellets.

Satiation procedure. Before test sessions, the rats were put in a distinctive consumption cage made of white plastic, measuring $21 \mathrm{~cm}$ wide $\times 31$ $\mathrm{cm}$ long $\times 19 \mathrm{~cm}$ high. The ceiling was a metal grid, through which a drinking spout could be inserted for presentation of sucrose solution. Cat litter was placed on the floor of the cage. When rats were to be sated on sucrose solution, $50 \mathrm{ml}$ was presented in a centrifuge tube fixed with a metal drinking spout and black rubber bung. When rats were to be sated on food pellets, $\sim 100 \mathrm{gm}$ of pellets was placed in a clear glass ramekin dish, and placed in the cage. The rat was given $90 \mathrm{~min}$ access to the reinforcer. The test session followed immediately. After each test session, the effect of the satiation procedure was assessed in a consumption test. The procedure used was identical to that used for pretest consumption but lasted only $30 \mathrm{~min}$.

Test procedure. There were two types of test session: single-lever and choice tests. In the single-lever test, there were 10 presentations of one of the levers, scheduled as in training, but no reinforcers were delivered. In the choice test, rats received 10 trials in which both levers were presented simultaneously. Again, no reinforcers were delivered. Retraining on the autoshaping procedure was given between each test. This consisted of two sessions, one with each reinforcer type. All rats received, in turn, pairs of single-lever tests after satiety with first one, and then the other reinforcer. These pairs of single-lever tests were conducted first with one lever, and then the other (i.e., a total of four tests). The arrangement of tests was fully counterbalanced with respect to order of reinforcer satiety and order of lever presentation. After this, all rats received choice tests after satiety with first one, and then the other reinforcer, again counterbalanced.

\section{Experiment 2: BLA lesions and devaluation in sensory preconditioning \\ Subjects}

The subjects were 15 male, hooded Lister rats with a mean free-feeding weight of $523 \mathrm{gm}$ (range, 425-600) at the beginning of the experiment. They had previously been used in a study of appetitive conditioning 
(experiment 1) (Blundell et al., 2001) but were naive to all aspects of the current stimuli and procedures. They were housed singly in a climatecontrolled vivarium (lights on 8 A.M. to 8 P.M.). The rats were tested during the light portion of the cycle. The rats were given ad libitum access to food throughout the experiment but were restricted to $30 \mathrm{~min}$ access to water at 11 A.M. and 5 P.M.

\section{Surgery and histology}

Eight of the rats received surgery to lesion the BLA, whereas seven had undergone the sham-surgery procedure. The surgical procedure was the same as that in experiment 1, except for the anesthetic that was used. Anesthesia was induced with $4 \%$ halothane, delivered in $\mathrm{O}_{2}$ and $\mathrm{N}_{2} \mathrm{O}$ gas $(\sim 1.0 \mathrm{l} / \mathrm{min}$ of each) in an induction chamber (IMS Ltd., Congleton, UK). When the rat was clearly anesthetized, the chamber was scavenged of halothane, and the rat's nose was placed into a facemask (IMS Ltd.), on a stereotaxic frame (Stoelting Inc.). Anesthesia was maintained with delivery of $1.5 \%$ halothane in $\mathrm{O}_{2}$ and $\mathrm{N}_{2} \mathrm{O}$ gas $(\sim 0.7 \mathrm{l} / \mathrm{min}$ of each), delivered through the facemask. The depth of anesthesia was monitored by assessing the pedal withdrawal reflex and responsivity to a mild tail pinch. The histological procedure used was identical to that used in experiment 1 .

\section{Apparatus}

Inverted $50 \mathrm{ml}$ centrifuge tubes equipped with stainless steel, ballbearing-tipped spouts were used to present measured amounts of flavor solution to the rats. The flavor stimuli that were used were $10 \%$ sucrose solution, $0.16 \mathrm{~m}$ saline solution, $0.01 \mathrm{~m}$ hydrochloric acid, and $60.0 \mu \mathrm{M}$ quinine (A, B, X, Y, respectively; solutions were counterbalanced in pairs $\mathrm{A}$ and $\mathrm{B}$, and $\mathrm{X}$ and $\mathrm{Y}$ ). When the solutions were presented in compound, they were formulated to retain these concentrations. All of the sessions were conducted in the rats' home cages.

\section{Procedure}

Preexposure. The rats were allowed $2 \mathrm{~d}$ to adjust to the water deprivation schedule before the start of phase one, which comprised 8 preexposure days. Rats received $30 \mathrm{~min}$ exposure to $10 \mathrm{ml}$ of the flavor compounds AX or BY, on alternate days, at 11 A.M. Half of the rats were exposed to AX on the first day, and half to BY. Thereafter, the solution the rats received was alternated. All rats were given free access to water for 30 min each day at 5 P.M.

Conditioning. At 11A.M. on day 1 of phase two, all of the rats were given $10 \mathrm{ml}$ of solution $\mathrm{X}$ for $30 \mathrm{~min}$, immediately followed by an injection of $0.15 \mathrm{M} \mathrm{LiCl}$ at $20 \mathrm{ml} / \mathrm{kg}(\mathrm{X}+)$. The rats were given $30 \mathrm{~min}$ free access to water at 5 P.M. and were allowed to recover on the following day, receiving $30 \mathrm{~min}$ access to water at 11 A.M. and 5 P.M. At 11 A.M. on day 3 of phase two, the rats were given $10 \mathrm{ml}$ of solution $\mathrm{Y}$ for $30 \mathrm{~min}$, with no consequence $(\mathrm{Y}-)$. They were given free access to water at 5 P.M., and the following day was a recovery day, identical to day 2 of phase two. This $4 \mathrm{~d}$ cycle was then repeated, so that each rat received two $\mathrm{X}+$ and two $\mathrm{Y}-$ training trials.

Test. Finally, all rats were tested for consumption of solutions A and B. They were given a two-bottle choice test, with $10 \mathrm{ml}$ of each solution available to the animals, for $30 \mathrm{~min}$, at 11 A.M. The side of presentation of each solution was counterbalanced. Consumption was measured by weighing the drinking bottles before and after the test.

\section{Results}

\section{Experiment 1: BLA lesions and devaluation of} autoshaped responding

The Pavlovian training procedure used in the present experiment was a version of autoshaping (or sign tracking) (Jenkins and Moore, 1973). When a retractable lever is presented to a rat, and the lever's retraction is paired with food reward, the rat will come to press that lever although there is no need for it to do so. The lever functions as a CS for food reward, and the rat will tend to approach and manipulate such a CS, often sniffing, licking, and biting the lever as though it were the US (Davey and Cleland, 1981). Previous work suggests that rats with BLA lesions show no deficit in the acquisition of autoshaped responding (Parkinson et

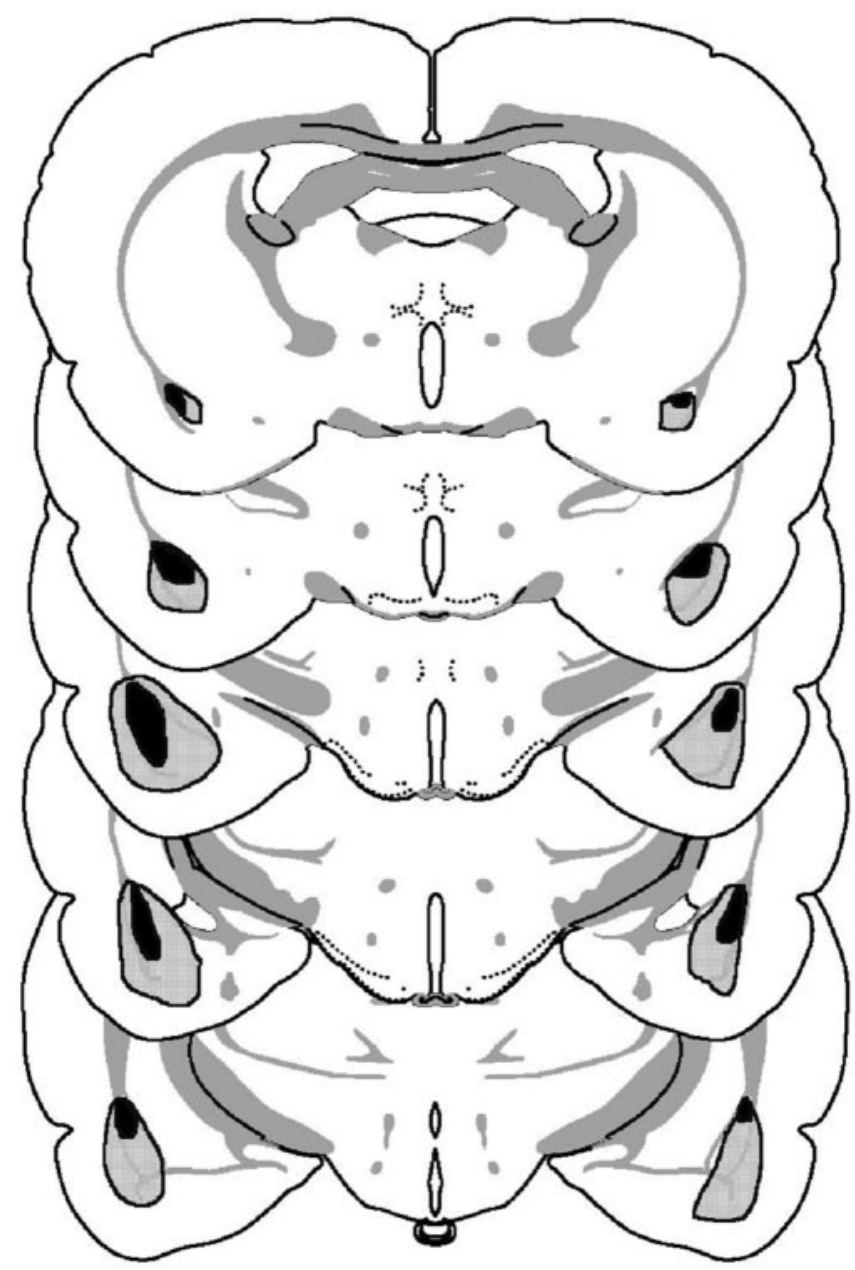

Figure 1. Schematic representation of excitotoxic lesions to the basolateral amygdala from experiment 1. The shaded areas represent the smallest (black) and largest (gray) extent of neuronal damage. Coronal sections are -1.8 to $-3.8 \mathrm{~mm}$ relative to bregma (Swanson, 1998).

al., 2000) but are no longer sensitive to post-training changes in the value of the reinforcer (Hatfield et al., 1996). The present experiment sought to replicate and extend these findings, examining choice behavior in an autoshaping task in which rats received separate presentations of two levers, each associated with a different reinforcer (food pellets or sucrose solution). Rats were then sated with one of the reinforcers, and the effect on responding in extinction was assessed. We might expect in normal animals that satiation with a particular reinforcer would selectively suppress responding on the lever associated with that reinforcer (Holland and Rescorla, 1975). The question of interest was whether animals with BLA lesions would show the same effect.

\section{Histology}

Three rats died during or immediately after surgery, two from the lesioned group and one sham-operated control. Histological analysis revealed that all rats in the lesioned group had bilateral lesions of the lateral and basolateral nuclei of the amygdala. Figure 1 shows the extent of the smallest and largest lesions that were included in the analysis. For some of the rats that were included in the experimental group, damage extended unilaterally into the basomedial nucleus. One rat also had damage to the central nucleus and was excluded from further analysis. There were five BLA-lesioned rats and seven sham-lesioned rats in the final analysis. 


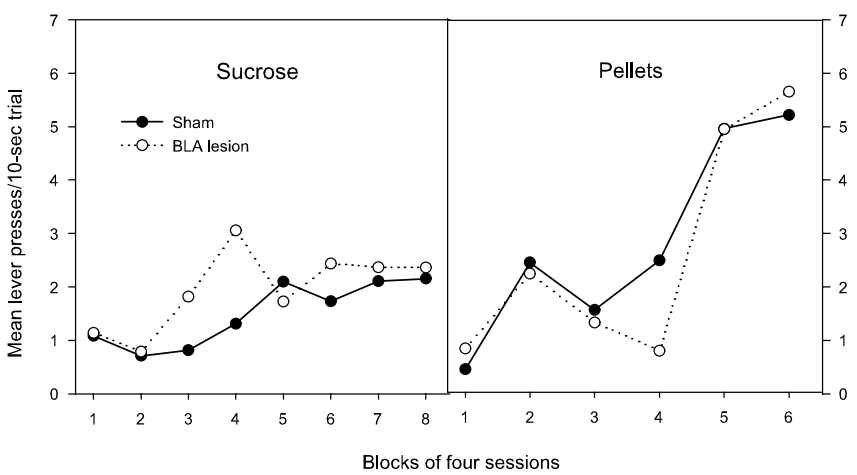

Figure 2. Group mean number of lever presses in each $10 \mathrm{sec}$ trial, during acquisition of autoshaping, with sucrose solution reward (left) and food pellet reward (right).

Acquisition of autoshaped responding. Figure 2 shows the group mean responses per trial for two-session blocks of initial acquisition of the autoshaped lever presses. Both BLA- and shamlesioned animals increased lever pressing over trials, and there was no obvious difference between the groups. Both BLA- and sham-lesioned animals made fewer responses when the reinforcer was sucrose than when the reinforcer was a food pellet. The data for the acquisition phase were separately analyzed for pellet reinforcer and sucrose reinforcer, because there were different numbers of training sessions. An $\alpha$ level of 0.05 was adopted for this and all subsequent statistical analyses.

An ANOVA was conducted on the data, summarized in the left panel of Figure 2 (acquisition with a sucrose solution US), with the between-groups variable of lesion (sham, BLA) and the within-subject variable of session (1-16). This revealed a significant effect of session $\left(F_{(15,204)}=3.67\right)$ but no significant effect of lesion $(F<1)$ and no interaction between these variables $(F<1)$.

A parallel ANOVA on the data, summarized in the right panel of Figure 2 (acquisition with food pellet US), yielded equivalent results. There was a significant main effect of session $\left(F_{(11,132)}=\right.$ 16.42) but no other significant effects $(F<1)$.

\section{Satiation}

During consumption tests assessing the efficacy of the satiety treatment, both BLA-lesioned and sham-lesioned rats consumed more of each type of reward when it was not the food on which they had been sated than when it was the food on which they had been sated. BLA-lesioned rats consumed ( \pm SEM) 7.9 (1.1) gm of the reward on which they had been sated, but 13.3 (1.3) gm of the reward on which they had not been sated. Similarly, sham-lesioned rats consumed ( \pm SEM) 3.9 (1.0) gm of the reward on which they had been sated, but 12.2 (1.1) gm of the reward on which they had not been sated. A two-way ANOVA conducted on the data from the sucrose consumption tests, with the variables of satiation (sated, nonsated) and lesion (BLA, sham), revealed a significant main effect of satiation $\left(F_{(1,10)}=41.98\right)$ but no effect of lesion $(F<1)$ and no lesion $\times$ satiation interaction $(F<1)$. An identical two-way ANOVA conducted on the data from the pellet consumption test revealed a significant main effect of satiation $\left(F_{(1,10)}=6.49\right)$, no main effect of lesion $\left(F_{(1,10)}=1.84\right)$, and no lesion $\times$ satiation interaction $(F<1)$.

\section{Single-lever tests}

Scores recorded on single-lever tests were pooled for each animal to produce a score for responding when the lever used on test was that associated with the reward on which the animal was sated (sated lever) and responding when the lever used on test was that associated with the reward on which the animal had not been sated (nonsated lever). Sham-lesioned rats made more responses on the nonsated lever than on the sated lever. Responses ( \pm SEM) made in extinction were: sated, 19.1 (6.9) lever presses (lp)/session; nonsated, 37.7 (13.1) lp/session. In contrast, BLA-lesioned rats showed a very much smaller difference in responding on the two levers during extinction. Responses ( \pm SEM) were: sated, 41.4 (10.1) lp/session; nonsated, 46.6 (13.1) lp/session. Hence, regardless of their state of specific satiety, BLA-lesioned rats responded during test at the same level as sham-lesioned rats on the nonsated lever. This was confirmed by a mixed ANOVA with factors of lesion (sham, BLA) and devaluation (sated, nonsated). Before analysis, scores were expressed as a percentage of baseline responding to reduced variability (baseline rates did not themselves differ: sham, $31.0 \mathrm{lp} / \mathrm{session;}$ BLA, $32.9 \mathrm{lp} / \mathrm{session;} t_{(10)}$ $<1.0$ ) and were subject to square-root transformation to reduce systematic changes in variance in relation to the mean (Howell, 1997). This analysis revealed a significant effect of devaluation $\left(F_{(1,10)}=15.3\right)$ and, more importantly, an interaction of lesion and devaluation $\left(F_{(1,10)}=6.5\right)$. Simple effects analysis of this interaction revealed a significant effect of lesion on devalued responding $\left(F_{(1,10)}=9.4\right)$, but not nondevalued responding $(F<$ $1)$, and a significant effect of devaluation in sham-lesioned animals $\left(F_{(1,10)}=25.1\right)$, but not BLA-lesioned animals $(F<1)$.

Magazine entries during the CS presentation were also recorded. The mean responses ( \pm SEM) were: sham: sated, 12.1 (4.8) entries/session; nonsated, 12.5 (3.7) entries/session; BLA: sated, 12.1 (2.8) entries/session; nonsated, 10.8 (2.6) entries/session. Hence, there was little effect of satiety on the number of magazine entries during the CS, and there was no difference between BLA- and sham-lesioned rats. This pattern of results was confirmed by a two-way ANOVA on the magazine-entry scores, which revealed no significant effects (all $F<1$ ).

\section{Choice test}

Once again, the responses on the sated lever and the on the nonsated lever were summed across tests. As might be expected, actual rates of responding $( \pm$ SEM) during the choice tests were lower than in single-lever tests [sham: sated, 3.0 (1.0) lp/session; nonsated, 12.6 (5.8) lp/session; BLA: sated, 6.5 (2.6) lp/session; nonsated, 6.1 (3.9) lp/session]. This is likely to reflect not only the effect of repeated extinction testing, but also a degree of generalization decrement in performance because of transfer from training with single levers to a test with both levers. Sham-lesioned rats made substantially more responses on the nonsated lever than on the sated lever. In contrast, BLA-lesioned rats showed no such effect of satiety on responding and, in fact, made slightly fewer responses on the nonsated lever than on the sated lever. Data were subject to square-root transformation before analysis by mixed ANOVA with factors of lesion and devaluation. This revealed an interaction of lesion and devaluation that approached the level of rejection of the null hypothesis $\left(F_{(1,10)}=4.6 ; p<0.06\right)$. Simple effects analysis, again, demonstrated a significant effect of devaluation in sham-lesioned animals $\left(F_{(1,10)}=9.8\right)$ but not BLAlesioned animals $(F<1)$. Because the levels of responding of the BLA-lesioned animals on the two levers lay between those of the sham-lesioned animals, simple effects analysis found no significant overall effect of lesion on either the sated or the nonsated lever (minimum, $F_{(1,10)}=2.3$ ). This also confirmed that lesions of the BLA did not alter absolute rates of lever pressing per se, but that levels of responding in single-lever and choice tests reflected a common absence of specific satiety devaluation that varied in nature depending on the availability of alternative responses. 


\section{Experiment 2: Sensory preconditioning}

Experiment 1 demonstrated that both BLA- and sham-lesioned rats could acquire autoshaped CRs, confirming the finding that lesions of the BLA do not impair acquisition of appetitive Pavlovian conditioning. When a specific US was devalued by prefeeding, sham-lesioned rats selectively reduced their extinction responding on the lever that had paired with that US during training. In contrast, BLA-lesioned rats demonstrated little of no influence of this manipulation. This pattern of results parallels that reported by Malkova et al. (1997) in primates and replicates a similar effect demonstrated by Hatfield et al. (1996), extending it to a procedure in which the US-devaluation is achieved without the use of taste-aversion learning (Morris et al., 1999).

Because satiety is reinforcer specific in normal animals, it must affect the representations of the unique sensory properties of the reinforcer (Balleine, 2001), a suggestion backed up by selective changes in specific hedonic responses to ingestion of a foodstuff across satiety (Berridge, 1991). Normal animals showed a selective reduction in lever pressing on the lever that had previously predicted delivery of the sated reinforcer, indicating that an association had formed between insertion of that lever into the Skinner box and the specific sensory properties of the consequent US. There are two possible accounts for the failure of sensitivity to devaluation in animals with lesions of the BLA. The first is that they are unable to use or modify representations conveying the current motivational value of associative outcomes. The second is that they are unable to learn about the sensory, incentive properties of motivationally significant events. These two accounts make different predictions about the effect of BLA lesions on devaluation in a sensory preconditioning task. If animals with BLA lesions are unable to access and modify associatively activated outcome representations, then we might expect that they would not be able to show evidence of outcome devaluation after sensory preconditioning. However, if BLA-lesioned rats are unable to learn about the sensory aspects of motivationally significant events (but have intact learning about neutral events), we would not expect a deficit in sensory preconditioning. Experiment 2 examined the effect of BLA lesions on sensory preconditioning.

\section{Histology}

Figure 3 shows the extent of the smallest and largest lesions. Figure 4 shows photomicrographs with representative sham and BLA lesions. Histological analysis revealed that all eight BLAlesioned rats had bilateral damage to both the basolateral and lateral nuclei of the amygdala. No rats were excluded.

Consumption during preexposure. All of the rats consumed similar amounts of fluid on all preexposure days. Mean consumptions ( \pm SEM) were: BLA AX, $9.2(0.3)$ ml; BLA BY, 9.2 (0.1) $\mathrm{ml}$; sham AX, $9.2(0.3) \mathrm{ml}$; sham BY, $9.4(0.1) \mathrm{ml}$. There were no obvious differences in the consumption of BLA-lesioned and sham-lesioned animals. A two-way ANOVA was conducted on these data with the variables of lesion (BLA, sham) and flavor (AX, BY). This found no significant effects (all $F<1$ ) or interactions, confirming that during the preexposure phase, there were no differences between volumes consumed by BLA-lesioned rats and sham-lesioned rats.

\section{Conditioning}

There were no obvious differences between sham-operated and BLA-lesioned animals in the acquisition of conditioned taste aversion to flavor X. The mean volumes consumed ( \pm SEM) during the first conditioning session were: sham X, $9.3(0.2) \mathrm{ml}$; sham

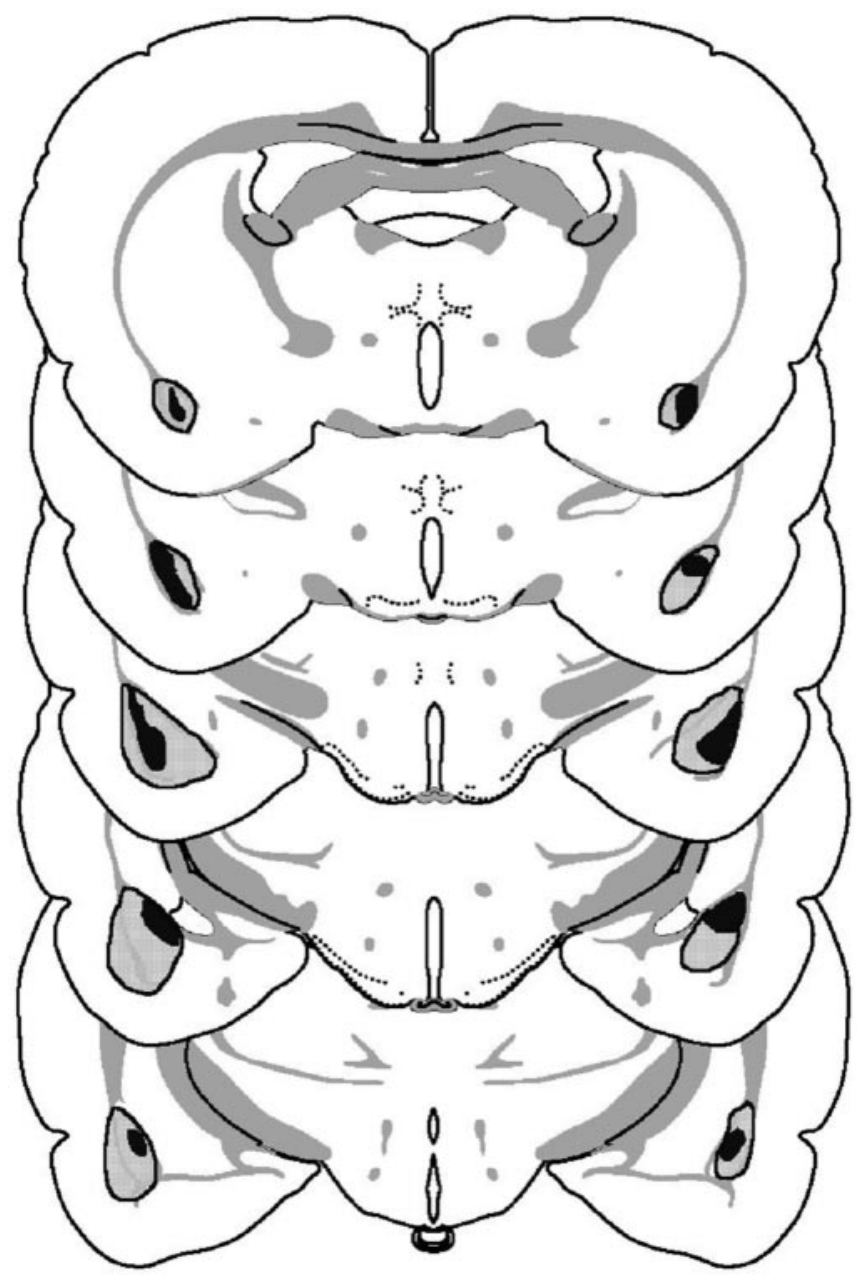

Figure 3. Schematic representation of excitotoxic lesions to the basolateral amygdala from experiment 2. Shaded areas represent the smallest (black) and largest (gray) extent of neuronal damage. Coronal sections are -1.8 to $-3.8 \mathrm{~mm}$ relative to bregma (Swanson, 1998).

Y, 8.7 (0.5) ml; BLA X, 9.1 (0.2) ml; BLA Y, 8.4 (0.5) ml. The mean volumes consumed ( \pm SEM) during the second conditioning sessions were: sham X, 3.4 (1.1) ml; sham Y, 9.1 (0.2) ml; BLA X, 3.6 (1.0) $\mathrm{ml}$; BLA Y, $8.8(0.2) \mathrm{ml}$. The reduction in consumption of $\mathrm{X}$ in the second conditioning session in the sham-lesioned animals reflects the acquisition of the conditioned taste aversion to $\mathrm{X}$, but not to Y. BLA-lesioned rats also reduced their consumption of X, appearing to be the same as the sham animals in their acquisition of the conditioned taste aversion. Both BLA- and sham-lesioned animals discriminated between flavors $\mathrm{X}$ and $\mathrm{Y}$, drinking more of flavor $\mathrm{Y}$ than flavor $\mathrm{X}$ in the second session. A three-way mixed ANOVA was conducted on these data, with variables of lesion (BLA, sham), flavor $(\mathrm{X}, \mathrm{Y})$, and session $(1,2)$. This revealed no significant main effect of lesion $(F<1)$, a significant main effect of flavor $\left(F_{(1,13)}=30.59\right)$, and a significant main effect of session $\left(F_{(1,13)}=47.39\right)$. The lesion $\times$ flavor interaction was not significant $(F<1)$, nor was that of lesion $\times$ session $(F<1)$. The flavor $\times$ session interaction was significant $\left(F_{(1,13)}=49.52\right)$, and the lesion $\times$ flavor $X$ session interaction was not significant $(F<$ $1)$. A simple main effects analysis of the flavor $\times$ session interaction revealed a significant effect of flavor at session $2\left(F_{(1,13)}=\right.$ $44.88)$ but not at session $1\left(F_{(1,13)}=4.62\right)$. There was also a significant effect of session on consumption of $\mathrm{X}\left(F_{(1,13)}=61.07\right)$ but not of $\mathrm{Y}\left(\mathrm{F}_{(1,13)}=1.05\right)$. This reflects the acquisition of an 

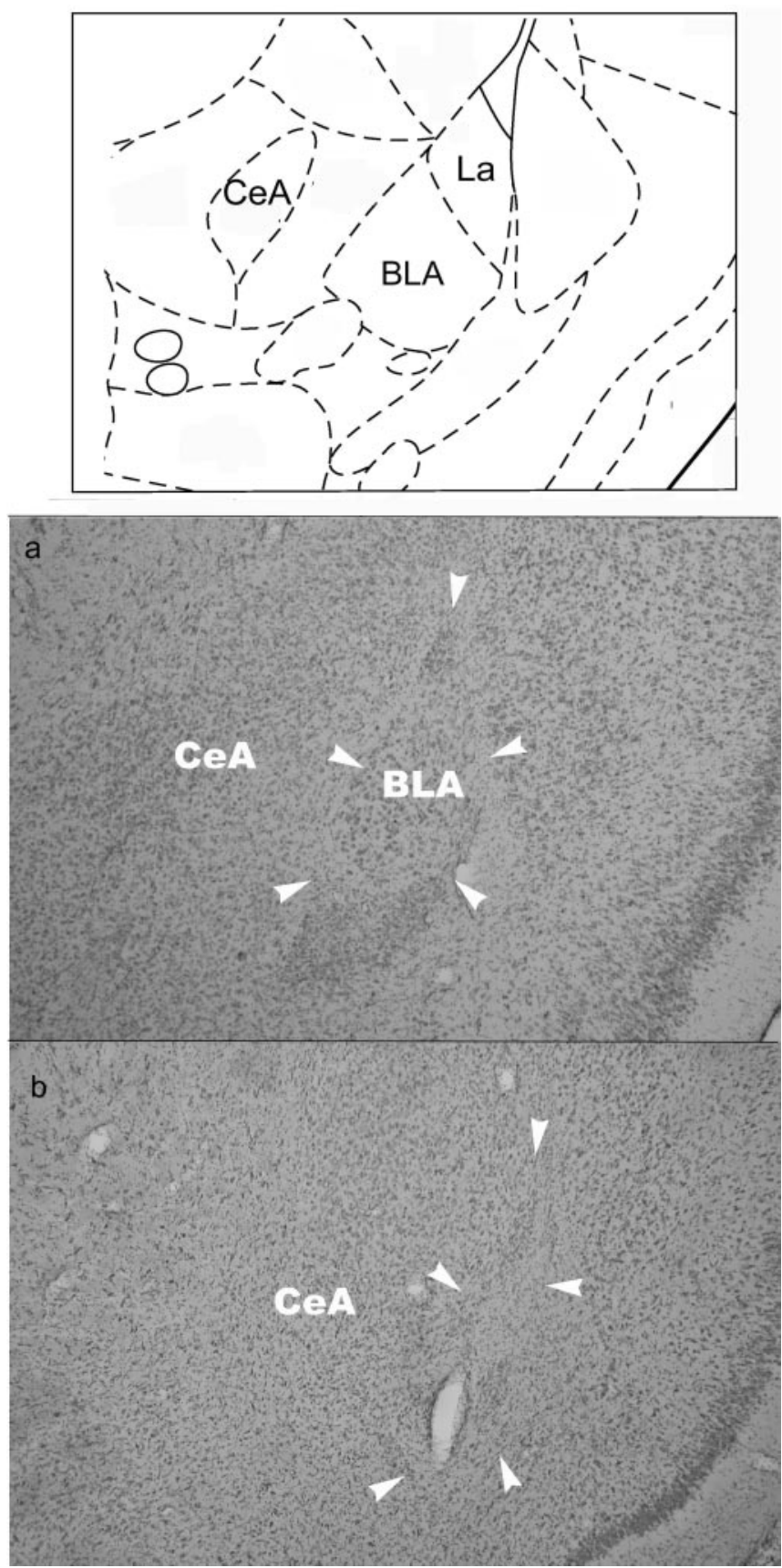

Figure 4. Photomicrographs from sections of two representative brains showing sham (a) and excitotoxic $(b)$ lesions basolateral nucleus of the amgydala. Each photomicrograph shows a high magnification of the area indicated in the associated outline, representing a section at 1.88 $\mathrm{mm}$ posterior to bregma. Subregions of the amygdala are marked (BLA, Basolateral nucleus; $L A$, lateral nucleus; $C \mathrm{C}$, central nucleus. In $a$, the BLA is marked by arrows; in $b$, the lesioned area is marked by arrows.

aversion to flavor X, but not flavor $\mathrm{Y}$, in session 2. The absence of significant effects involving lesion confirms that the BLA lesion did not affect conditioned taste aversion learning in these animals.

\section{Test}

The mean volumes ( \pm SEM) of $\mathrm{A}$ and $\mathrm{B}$ consumed by BLA and sham-lesioned rats during the test session were: sham A, 6.5 (1.5) ml; sham B, 8.8 (0.6) ml; BLA A, 5.4 (1.6) ml; BLA B, 9.3 (0.6) ml. Hence, both BLA- and sham-lesioned rats drank less of solution
A than of solution B; if anything, the aversion to A was slightly more marked in the BLA-lesioned animals. A two-way mixed ANOVA was conducted on these data, with the variables of lesion (BLA, sham) and flavor (A, B). This revealed a significant main effect of flavor $\left(F_{(1,13)}=4.94\right)$, no significant main effect of lesion $(F<1)$, and no lesion $\times$ flavor interaction $(F<1)$. Thus, both BLA- and sham-lesioned rats showed a sensory preconditioning effect, drinking less of a flavored solution that had previously been paired with a flavor to which they had developed an aversion, than of a flavored solution that had been paired with a neutral flavor.

\section{Discussion}

Experiment 1 confirmed that rats with BLA lesions show normal acquisition of appetitive Pavlovian conditioning. Both sham- and BLA-lesioned animals acquired autoshaped lever pressing with both sucrose and food pellet rewards. A satiety test was carried out to examine the BLA-lesioned rats' sensitivity to reinforcer devaluation. The CRs of the BLA-lesioned rats were insensitive to sensory-specific satiety, in both single-lever and choice tests, but a consumption test confirmed the success of the satiety treatment itself. This complements previous findings (Hatfield et al., 1996; Parkinson et al., 2000; Balleine et al., 2003), extending the results to satiety-induced devaluation in an autoshaping procedure that provided both single stimulus and choice tests of Pavlovian approach CRs.

Experiment 2 sought to determine whether BLA-lesioned animals have a general deficit in adjusting responding according to the current motivational value of an associatively activated outcome representation. Rats were presented with two compound flavor stimuli, each made up of two different flavors. Subsequently, one of these flavors was devalued by pairing its consumption with nausea-inducing injection of LiCl. Both BLA- and sham-lesioned rats acquired the conditioned flavor aversion and also subsequently rejected the neutral flavor that had previously been paired with this newly devalued flavor. That is, they showed no deficit in outcome devaluation in a sensory preconditioning task, demonstrating that BLA-lesioned rats can alter their responding in accordance with the current motivational value of an associatively retrieved outcome representation.

Comparison of the designs of experiments 1 and 2 reveals that they are very similar in structure. In experiment 1, a CS was paired with a US, which in turn was devalued; lesions of the BLA interfered with expression of the devaluation in performance to the CS. In experiment 2, flavor A was paired with flavor X, which in turn was devalued; lesions of the BLA were without effect on expression of the devaluation in performance to flavor A. Comparing parameters in experiments 1 and 2, both the CS and US and flavors $\mathrm{A}$ and $\mathrm{X}$ were novel. The methods of devaluation (specific satiety and injection of $\mathrm{LiCl}$ ) were different, but Hatfield et al. (1996) have demonstrated devaluation effects in appetitive Pavlovian conditioning similar to those found in experiment 1 using $\mathrm{LiCl}$ injection as the method of devaluation, suggesting that this is not the source of the differential involvement of the BLA. The assessed CRs were different (consumption in experiment 2 and Pavlovian approach behavior in experiment 1). However, current theories of BLA function do not suggest that this difference could account for the differential sensitivity to BLA lesions of changes in stimulus value between the two experiments, and deficits in devaluation have also been found with measures of instrumental lever pressing (Balleine et al., 2003) magazine approach (Hatfield et al., 1996) and reaching in primates (Malkova et al., 1997). Nor were the lesions in experiment 2 simply ineffec- 
tive, because these same animals were previously used in experiment 1 of Blundell et al. (2001), which found dramatic lesion effects on sensitivity to the nature of reward in a differential outcomes procedure.

One interpretation of these contrasting results is that BLA lesions are without effect on neutral S-S learning. Hence, performance at test in experiment 2 is mediated by intact S1-S2 associations in both sham and lesioned animals. This complements recent findings suggesting that $\mathrm{S}-\mathrm{S}$ associations in second-order conditioning are intact after BLA lesions (Setlow et al., 2002) and extends these findings to demonstrate that despite total BLA damage, animals can remain sensitive to outcome devaluation. Crucially, BLA-lesioned animals must still be sensitive to postlesion changes in the value of the associatively activated representation of $\mathrm{S} 2$ to show appropriate devaluation-induced changes in performance to $\mathrm{S} 1$. Although this rejects suggestions that the BLA is necessarily required for processing information about the acquired motivational value of otherwise neutral stimuli, why might an S1-S2 associative structure result in intact devaluation performance when a parallel CS-US associative structure does not?

One plausible account of the current data is that the BLA is only part of a wider limbic-cortico-striatal circuitry responsible for the representation of rewards. That is, there are extraamygdala representations of associative outcomes that are capable of representing and updating their current motivational value. A substantial body of work has highlighted the role of connectivity between the amygdala, prefrontal cortex, and nucleus accumbens in the development of such representations (Balleine et al., 2003). Of particular relevance are findings that cells within the orbitofrontal cortex of primates respond to reward delivery (and signals for reward delivery) in accordance with the current motivational state of the animal (Rolls, 2000) and evidence that lesions of the gustatory cortex (Balleine and Dickinson, 2000), nucleus accumbens core (Corbit et al., 2001), or ventrolateral prefrontal cortex (Gallagher et al., 1999) produce deficits in reward devaluation. However, even with this distributed reward representation system, one must still account for the failure of BLA-lesioned animals to remain sensitive to changes in outcome value in first-order conditioning (experiment 1) (Balleine et al., 2003; Hatfield et al., 1996).

Recent work (Blundell et al., 2001; Killcross and Blundell, 2002; Balleine et al., 2003) has suggested that the BLA is specifically involved in encoding the sensory-specific aspects of motivationally significant stimuli and, hence, in associative processes that allow arbitrary stimuli or responses access to the incentive or hedonic properties of their associated outcomes. By this account, acquisition of appetitive-conditioned responding in BLAlesioned animals is mediated by $\mathrm{S}-\mathrm{R}$ reinforcement processes (Setlow et al., 2002) that are not dependent on incentive representations of outcomes (and, hence, are independent of BLA function). We also suggested that the target response of this S-R relation is unlikely to be a highly specified behavioral response, but is instead a generalized emotional response, and further that there is little theoretical difference between the acquisition of a generalized emotional S-R association, and the acquisition of stimulus-outcome associations in which the outcome is represented only in terms of the generalized motivational properties of the reinforcer and not its specific sensory aspects (Konorski, 1967; Hall, 1996). That some such general reinforcement process is intact in BLA-lesioned animals is suggested by their acquisition of first-order Pavlovian (experiment 1) (Setlow et al., 2002) and instrumental conditioning (Blundell et al., 2001; Balleine et al., 2003) and their ability to show normal conditioned elevation
(Blundell et al., 2001; Holland and Gallagher, 2003) and suppression (Killcross et al., 1997). Although this general reinforcement process is sufficient to mediate first-order CRs such as Pavlovianconditioned approach, US devaluation impacts primarily on the hedonic, sensory representation (Berridge, 1996, 2001; Balleine, 2001), and BLA-lesioned rats, therefore, fail to show any effect of the devaluation (cf. experiment 1 ). Note that BLA lesions do not impair the direct perception of palatability, as shown by BLAlesioned animals' preserved ability to reject the sated food in a consumption test; rather, it is associatively activated representations of the sensory aspects of the reinforcer that are disrupted. Because it is only representations of the sensory aspects of motivationally significant outcomes that are disrupted, this hypothesis also accounts for the results of experiment 2 . In sensory preconditioning, representation of neutral S1-S2 associations is not dependent on the BLA (Nicholson and Freeman, 2000), and, following the argument above, the subsequent change in the hedonic evaluation of the initially neutral associative outcome S2 is represented elsewhere in distributed limbic-cortico-striatal circuitry. Hence, devaluation performance after BLA lesions is normal. The critical difference between this and first-order conditioning is that the motivational significance of the first-order US obliges BLA-based processing of the US, revealing deficits in representation of the sensory-specific aspects of this motivationally significant outcome.

In fact, the more conservative hypothesis that the BLA is responsible for the representation of the sensory properties of outcomes, regardless of their motivational salience, could also provide an explanation of these results. If we assume, contrary to the position above, that there is no representational difference between motivationally significant and neutral outcomes (for example, although the flavors presented in experiment 2 were neutral, they were presented in aqueous solution to thirsty animals), then a crucial difference could be that experiment 1 required formation of visual-taste (lever-food) associations, whereas experiment 2 required taste-taste associations. Failures in devaluation might, therefore, be because of deficits in cross-modal association rather than in reward representation. Although this hypothesis still requires that the BLA is responsible for associations involving the sensory, hedonic aspects of the US (i.e., the selective taste components of the reward), this explanation would be in line with previous interpretations of BLA function (Hatfield et al., 1992) and suggests that deficits in devaluation in first-order conditioning, and in taste-potentiated odor aversion, have the same fundamental cause: failure to form, or make use of, arbitrary cross-modal stimulus-taste associations. Although this presents an appealing simplification of data examining the effects of BLA damage, there is reason to be cautious. Setlow et al. (2002) have recently suggested that second-order rearing CRs mediated by cross-modal (auditory-visual) S-S associations were intact after BLA lesions made before first-order conditioning. This may provide evidence for intact cross-modal acquisition after BLA damage, although the presence of these associations was inferred rather than explicitly demonstrated. Similarly, recent studies in monkeys (Malkova and Murray, 1996; Goulet and Murray; 2001) have failed to find deficits in established cross-modal tactualvisual-delayed nonmatching-to-sample after selective amygdala damage, although these studies do not directly examine the acquisition of arbitrary cross-modal associations.

In summary, we have demonstrated that BLA-lesioned animals can remain sensitive to post-training changes in the motivational value of outcomes. We have suggested (Blundell et al., 2001; Balleine et al., 2003) that BLA lesions selectively disrupt 
encoding the sensory-specific aspects of motivationally significant stimuli and do not impact on the representation of the general reinforcing aspects of outcomes. Hence, devaluation in sensory preconditioning makes use of neural systems independent of the BLA, and performance is intact. Because these sensory aspects of the reinforcer appear to play an important role in the hedonic evaluation of rewards and their palatability (Berridge, 1996; Balleine, 2001), it appears that the involvement of the BLA in reward-related processes is best characterized as reflecting aspects of liking rather than wanting (Simbayi et al., 1986; Berridge, 1996; Killcross and Blundell, 2002).

\section{References}

Balleine BW (2001) Incentive processes in instrumental conditioning. In: Handbook of contemporary learning theories (Mowrer RR, Klein SB, eds), pp 307-366. Hillsdale, NJ: Erlbaum.

Balleine BW, Dickinson A (2000) Effect of lesions of the insular cortex on instrumental conditioning: evidence for a role in incentive memory. J Neurosci 20:8954-8964.

Balleine BW, Killcross AS, Dickinson A (2003) The effect of lesions of the basolateral amygdala on instrumental conditioning. J Neurosci 23:666-675.

Berridge KC (1991) Modulation of taste affect by hunger, caloric satiety, and sensory-specific satiety in the rat. Appetite 16:103-120.

Berridge KC (1996) Food reward: brain substrates of wanting and liking. Neurosci Biobehav Rev 20:1-25.

Berridge KC (2001) Reward learning: reinforcement, incentives, and expectations. In: The psychology of learning and motivation—advances in research and theory, Vol 40 (Medin DL, ed), pp 223-278. San Diego: Academic.

Blundell PJ, Hall G, Killcross AS (2001) Lesions of the basolateral amygdala disrupt selective aspects of reinforcer representation in rats. J Neurosci 21:9018-9026.

Corbit L, Muir J, Balleine BW (2001) The role of the nucleus accumbens in instrumental conditioning: evidence for a functional dissociation between accumbens core and shell. J Neurosci 21:3251-3260.

Davey GCL, Cleland GG (1981) Topography of signal-centered behavior in the rat: effects of deprivation state and reinforcer type. J Exp Anal Behav 38:291-304.

Dunn LT, Everitt BJ (1988) Double dissociations of the effects of amygdala and insular cortex lesions on conditioned taste-aversion, passiveavoidance, and neophobia in the rat using the excitotoxin ibotenic acid. Behav Neurosci 102:3-23.

Gallagher M, McMahan RW, Schoenbaum G (1999) Orbitofrontal cortex and representation of incentive value in associative learning. J Neurosci 19:6610-6614.

Goulet S, Murray EA (2001) Neural substrates of crossmodal association memory in monkeys: the amygdala versus the anterior rhinal cortex. Behav Neurosci 115:271-284.

Hall G (1996) Learning about associatively activated stimulus representations: implications for acquired equivalence and perceptual learning. Anim Learn Behav 24:233-255.

Hatfield T, Graham PW, Gallagher M (1992) Taste-potentiated odor aver- sion learning: role of the amygdaloid basolateral complex and central nucleus. Behav Neurosci 106:286-293.

Hatfield T, Han JS, Conley M, Gallagher M, Holland P (1996) Neurotoxic lesions of basolateral, but not central, amygdala interfere with Pavlovian second-order conditioning and reinforcer devaluation effects. J Neurosci 16:5256-5265.

Holland PC, Gallagher M (2003) Double dissociation of the effects of lesions of basolateral and central amygdala on conditioned-stimulus potentiated feeding and Pavlovian-instrumental transfer. Eur J Neurosci 17:1680-1694.

Holland PC, Rescorla RA (1975) Second-order conditioning with food unconditioned stimulus. J Comp Physiol Psychol 88:459-467.

Howell DC (1997) Statistical methods for psychology, Ed 5. Belmont, CA: Wadsworth.

Jenkins HM, Moore BR (1973) The form of the auto-shaped response with food or water reinforcers. J Exp Anal Behav 20:163-181.

Killcross AS, Blundell P (2002) Associative representations of emotionally significant outcomes. In: Emotional cognition (advances in consciousness research) (Moore S, Oaksford M, eds), pp 35-73. Amsterdam: Benjamins.

Killcross AS, Everitt BJ, Robbins TW (1997) Different types of fear-related behaviour mediated by separate nuclei within amygdala. Nature 388:377-380.

Konorski J (1967) Integrative activity of the brain: an interdisciplinary approach. Chicago: University of Chicago.

Malkova L, Murray EA (1996) Effects of partial versus complete lesions of the amygdala on cross-modal associations in cynomolgus monkeys. Psychobiology 24:255-264.

Malkova L, Gaffan D, Murray EA (1997) Excitotoxic lesions of the amygdala fail to produce impairment in visual learning for auditory secondary reinforcement but interfere with reinforcer devaluation effects in rhesus monkeys. J Neurosci 17:6011-6020.

Morris R, Frey S, Kasambira T, Petrides M (1999) Ibotenic acid lesions of the basolateral, but not the central, amygdala interfere with conditioned taste aversion: evidence from a combined behavioral and anatomical tract-tracing investigation. Behav Neurosci 113:291-302.

Nicholson DA, Freeman Jr JH (2000) Lesions of the perirhinal cortex impair sensory preconditioning in rats. Behav Brain Res 112:69-75.

Parkinson JA, Robbins TW, Everitt BJ (2000) Dissociable roles of the central and basolateral amygdala in appetitive emotional learning. Eur J Neurosci 12:405-413.

Rizley RC, Rescorla RA (1972) Associations in second-order conditioning and sensory preconditioning. J Comp Physiol Psychol 81:1-11.

Rolls ET (2000) The orbitofrontal cortex and reward. Cereb Cortex 10: $284-294$.

Setlow B, Gallagher M, Holland PC (2002) The basolateral complex of the amygdala is necessary for acquisition but not expression of CS motivational value in appetitive Pavlovian second-order conditioning. Eur J Neurosci 15:1841-1853.

Simbayi LC, Boakes RA, Burton MJ (1986) Effects of basolateral amygdala lesions on taste aversions produced by lactose and lithium chloride in the rat. Behav Neurosci 100:455-465.

Swanson LW (1998) Brain maps: structure of the rat brain. Amsterdam: Elsevier. 